\title{
A Mixed Normalization Capacitance Model for Electrical Capacitance Tomography
}

\author{
Lifeng Zhang ${ }^{\mathrm{a}}$, Feng Zhu ${ }^{\mathrm{b}}$, Qin Zhang ${ }^{\mathrm{c}}$ \\ Department of Automation, North China Electric Power University, Baoding 071003, China

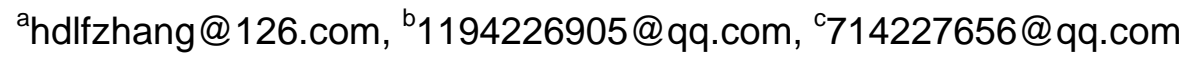

Keywords: Electrical resistance tomography, image reconstruction, capacitance normalization.

\begin{abstract}
Electrical capacitance tomography (ECT) is a technique developed in recent 30 years, which aims to measure multi-phase flow. The image reconstruction for ECT is an inverse problem to find the permittivity distribution in a pipe. The normalized capacitance measurements are needed during the process of image reconstruction. Parallel normalization model is the most commonly used normalization model, and series normalization model was also studied by some researchers. In this paper, a mixed normalization model based on electrical field center line (EFCL) and multi-threshold method was presented. The sensitivity matrices based on different normalization models were obtained and image reconstruction was carried out accordingly. Simulation and static experiment indicate that reconstructed images with higher quality can be obtained based on the presented model.
\end{abstract}

\section{Introduction}

Electrical capacitance tomography (ECT) is one kind of process tomography (PT) techniques which was developed during the late 1980s [1]. The task of image reconstruction for ECT is to determine the permittivity distribution of material over the cross-section using the measured capacitances.

Image reconstruction algorithms of ECT can usually be divided into two classes [2]. One is non-iterative algorithm and the other is iterative algorithm. The sensitivity matrix and normalization capacitances are needed for all the algorithms of both classes. The commonnly used normalization capacitance model is parallel normalization model, and another approach considers series normalization model [3]. Recently, the normalization capacitance model based on the distance between electrical field centre lines (EFCL) of each electrode pairs and perturbed element was presented [4].

In this paper, a mixed normalization capacitance model was presented, and the seneitivity matrix was calculated based on EFCL and multi-threshold. Image reconstruction was carried out using Landweber iterative algorithm based on the mixed normalization model. Compared with parallel and series normalization models, the image quality has been improved using the mixed normalization mode.

\section{Principle of ECT}

Mathematical Model. ECT technique involves a number of capacitive electrodes mounted circumferentially around a flow pipe. ECT is composed of forward problem and inverse problem. The forward problem is to calculate the potential distribution for a known distribution of dielectric constant, and then to determine the capacitance measurements. The inverse problem is also called image reconstruction.

The electrical field inside an ECT sensor can be calculated using the Laplace equation which is given by

$$
\nabla \cdot[\varepsilon(x, y) \nabla u(x, y)]=0
$$


where $\varepsilon(x, y)$ is the permittivity distribution in the sensing field, $u(x, y)$ is the potential distribution. In general, it is impossible to solve (1) for an inhomogeneous permittivity distribution analytically. The potential $u(x, y)$ can be calculated using finite element method (FEM).

The relationship between capacitance $C$ and $\varepsilon(x, y)$ is governed by the following equation

$C=\frac{Q}{V}=-\frac{1}{V} \oint_{\Gamma} \varepsilon(x, y) \nabla u(x, y) d \Gamma$

where $Q$ is the charge of an electrode, $V$ is the potential difference between an electrode pair and $\Gamma$ is the electrode surface.

Calculation of Sensitivity Matrix. The linearized and discrete form of (2) based on FEM calculation can be expressed as

$\lambda_{N \times 1}=\mathbf{S}_{N \times M} \mathbf{G}_{M \times 1}$

where $\lambda$ is the normalized capacitance vector, $\boldsymbol{G}$ is the normalized permittivity vector, $\boldsymbol{S}$ is the sensitivity matrix of normalized capacitance with respect to normalized permittivity, $N$ is the number of independent measurements and $M$ is the number of elements used in FEM.

When the parallel normalization model (PM) is used, the normalized capacitance of electrode pair $i-j$ can be defined as

$$
\lambda_{i j}^{P}=\frac{C_{i j}^{m}-C_{i j}^{l}}{C_{i j}^{h}-C_{i j}^{l}}
$$

The normalized capacitance is a linear function of measurement capacitance. The sensitivity matrix $\boldsymbol{S}$ is defined as

$$
\mathbf{S}_{i j}(k)=\mu(k) \cdot \frac{\mathbf{C}_{i j}^{m}(k)-\mathbf{C}_{i j}^{l}}{\mathbf{C}_{i j}^{h}-\mathbf{C}_{i j}^{l}} \cdot \frac{1}{\varepsilon_{h}-\varepsilon_{l}}
$$

where $\varepsilon_{h}$ and $\varepsilon_{l}$ are high and low permittivity values, $\mathbf{C}_{i j}^{h}$ and $\boldsymbol{C}_{i j}^{l}$ represent capacitance of electrode pair $i-j$ when the pipe is full of high and low permittivity material, respectively. $C_{i j}^{m}$ is the measurement capacitance of electrode pair $i-j$ when the $k$ th element has high permittivity and the other elements have low permittivity. $\mu(k)$ is a correction factor related to the area of the $k$ th in-pipe element.

While for the series normalization model (SM), the normalized capacitance of electrode pair $i-j$ is a nonlinear function of measurement capacitance, and is defined as

$$
\lambda_{i j}^{S}=\frac{1 / \boldsymbol{C}_{i j}^{m}-1 / \boldsymbol{C}_{i j}^{l}}{1 / \boldsymbol{C}_{i j}^{h}-1 / \boldsymbol{C}_{i j}^{l}}
$$

The sensitivity matrix of series normalization model can be calculated according to (7)

$$
\boldsymbol{S}_{i j}(k)=\mu(k) \cdot \frac{1 / \boldsymbol{C}_{i j}^{m}(k)-1 / \boldsymbol{C}_{i j}^{l}}{1 / \boldsymbol{C}_{i j}^{h}-1 / \boldsymbol{C}_{i j}^{l}} \cdot \frac{1}{\varepsilon_{h}-\varepsilon_{l}}
$$

In fact, neither parallel nor series normalization models can represent all the real permittivity distributions correctly. It seems that it is a better method to find a mixture of two models.

The EFCL between electrode pair 1-5 for a 12-electrode ECT sensor is shown in Fig. 1 and $d(k)$ is the distance between the $k$ th element and the EFCL.

To combine the parallel and series normalization models, the coefficients will be assigned to each model. The mixed normalization model (MM) for capacitance of electrode pair $i-j$ is expressed as

$$
\lambda_{i j}^{C}=(1-\alpha)\left(\frac{\boldsymbol{C}_{i j}^{m}-\boldsymbol{C}_{i j}^{l}}{\boldsymbol{C}_{i j}^{h}-\boldsymbol{C}_{i j}^{l}}\right)+\alpha\left(\frac{1 / \boldsymbol{C}_{i j}^{m}-1 / \boldsymbol{C}_{i j}^{l}}{1 / \boldsymbol{C}_{i j}^{h}-1 / \boldsymbol{C}_{i j}^{l}}\right)
$$

where $\alpha$ is the coefficient of the mixed normalization capacitance model. 


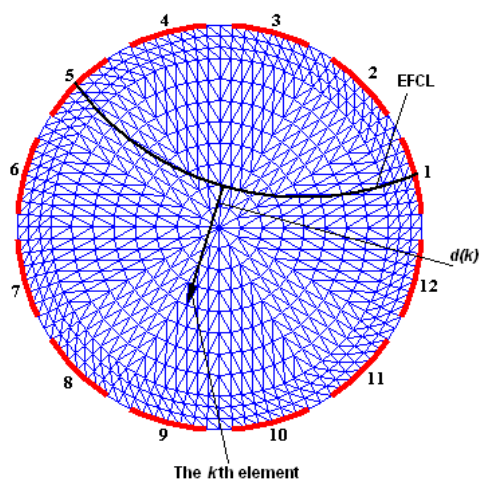

Fig.1 EFCL of electrode pair 1-5

The sensitivity matrix of mixed normalization model is defined as

$\mathbf{S}_{i j}(k)=\mu(k) \cdot\left[(1-\beta(k))\left(\frac{\mathbf{C}_{i j}^{m}(k)-\mathbf{C}_{i j}^{l}}{\mathbf{C}_{i j}^{h}-\mathbf{C}_{i j}^{l}}\right)+\beta(k)\left(\frac{1 / \mathbf{C}_{i j}^{m}(k)-1 / \mathbf{C}_{i j}^{l}}{1 / \mathbf{C}_{i j}^{h}-1 / \mathbf{C}_{i j}^{l}}\right)\right] \cdot \frac{1}{\varepsilon_{h}-\varepsilon_{l}}$

$\beta(k)$ is defined as the coefficient of the $k$ th element. The value of $\beta(k)$ is used to calculate the sensitivity matrix and it is determined by $d(k)$ and the multi-threshold. In this paper, the inner diameter of pipe is $120 \mathrm{~mm}$ and it is divided into 11 segments, and a mesh with 768 triangular elements is used in FEM calculation.

Thus, we have

$\beta(k)=f(d(k))=1-0.1 i \quad$ if $11 i \leq d(k)<11(i+1) \quad(i=0,1, \mathrm{~L}, 10 ; k=1,2, \mathrm{~L}, 768)$

Landweber Algorithm Based on Mixed Normalization Capacitance Model. Landweber iterative algorithm can be rewritten as [5]

$\boldsymbol{G}_{k+1}=\boldsymbol{G}_{k}+\eta_{k} \boldsymbol{S}^{T}\left(\lambda^{C}-\boldsymbol{S G}_{k}\right)$

$\mathbf{e}_{k+1}=\boldsymbol{\lambda}^{C}-\mathbf{S G}_{k+1}=\boldsymbol{\lambda}^{C}-\mathbf{S}\left(\mathbf{G}_{k}+\eta_{k} \mathbf{S}^{T} \mathbf{e}_{k}\right)=\mathbf{e}_{k}-\eta_{k} \mathbf{S S}^{T} \mathbf{e}_{k}=\left(\mathbf{I}_{N}-\eta_{k} \mathbf{S S}^{T}\right)\left[\left(1-\alpha_{k}\right) \lambda^{P}+\alpha_{k} \lambda^{S}-\mathbf{S G}_{k}\right]$

The norm of $\boldsymbol{e}_{k+1}$ is

$\mathbf{J}(\eta, \alpha)=\left\|\mathbf{e}_{k+1}\right\|^{2}=\left\|\left(\mathbf{I}_{M}-\eta_{k} \mathbf{S S}^{T}\right)\left[\lambda^{P}+\alpha_{k}\left(\lambda^{S}-\lambda^{P}\right)-\mathbf{S G}_{k}\right]\right\|^{2}$

Where $N$ is the number of independent measurements. Let $\frac{\partial \boldsymbol{J}(\eta, \alpha)}{\partial \eta}=0$ and $\frac{\partial \boldsymbol{J}(\eta, \alpha)}{\partial \alpha}=0$, we have

$$
\begin{aligned}
& \eta_{k}=\frac{\left\|\boldsymbol{S}^{T}\left[\lambda^{P}+\alpha_{k}\left(\lambda^{S}-\lambda^{P}\right)-\boldsymbol{S} \boldsymbol{G}_{k}\right]\right\|^{2}}{\left\|\boldsymbol{S} \boldsymbol{S}^{T}\left[\lambda^{P}+\alpha_{k}\left(\lambda^{S}-\lambda^{P}\right)-\boldsymbol{S} \boldsymbol{G}_{k}\right]\right\|^{2}} \\
& \alpha_{k}=\frac{\boldsymbol{A}^{T} \boldsymbol{B}^{T} \boldsymbol{B} \boldsymbol{S} \boldsymbol{G}_{k}+\left(\boldsymbol{A}^{T} \boldsymbol{B}^{T} \boldsymbol{B} \boldsymbol{S} \boldsymbol{G}_{k}\right)^{T}-\boldsymbol{A}^{T} \boldsymbol{B}^{T} \boldsymbol{B} \boldsymbol{\lambda}^{P}-\left(\boldsymbol{A}^{T} \boldsymbol{B}^{T} \boldsymbol{B} \boldsymbol{\lambda}^{P}\right)^{T}}{2 \boldsymbol{A}^{T} \boldsymbol{B}^{T} \boldsymbol{B} \boldsymbol{A}}
\end{aligned}
$$

Where $\boldsymbol{A}=\lambda^{S}-\lambda^{P}$ and $\boldsymbol{B}=\boldsymbol{I}_{M}-\eta_{k} \boldsymbol{S} \boldsymbol{S}^{T}$.

\section{Simulation and Static Experiments}

Simulation Experiment. Comparison is carried out with the parallel, series and mixed normalization models using Landweber iterative algorithm. Three permittivity distributions and the reconstructed images at the 200th iteration using PM, SM and MM are shown in Fig. 2, respectively.

Static Experiment. To verify the performance of the presented model, static experiment was also carried out using a FPGA-based ECT system [6].

To facilitate visual observations of the flow regime, the horizontal test section (0.6 $\mathrm{m}$ long) is a Perspex pipe (100 $\mathrm{mm}$ in diameter). The material used to stimulate each flow regime is nylon plastic pellets. Two true distributions were tested and the reconstructed images using Landweber iterative algorithm at 200th iteration for PM, SM and MM can be seen in Fig. 3.

From Figs. 2 and 3, it can be seen clearly that for these two complicated flow regimes, the reconstructed images using PM cannot be accepted. Compared SM with MM, the shapes of 
reconstructed images using $\mathrm{MM}$ are more close to the real distributions that that of SM. And objects can be separated more clearly using MM.

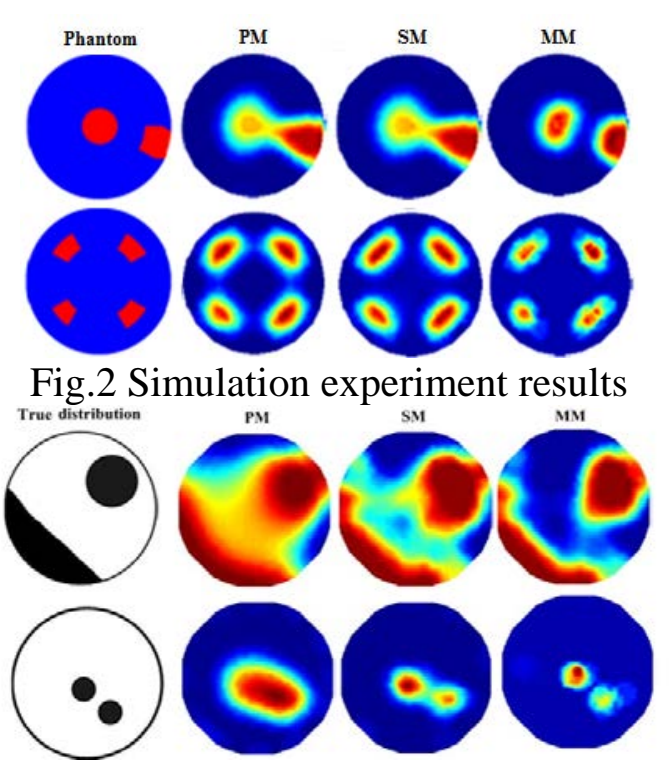

Fig. 3 Static experiment results

\section{Summary}

In this paper, PM, SM and MM are compared. The calculation of sensitivity matrix of MM based on EFCL and a multi-threshold scheme was proposed and Landweber iterative algorithm based on MM was also presented. Simulation and static experiment results show that the reconstructed images with MM have superior image quality than those with PM and SM.

\section{Acknowledgments}

The author wishes to thank the support of the National Natural Science Foundation of China (51306058) and the Fundamental Research Funds for the Central Universities (2014MS142).

\section{References}

[1] W. Warsito and L. -S. Fan, Measurement of real-time flow structures in gas-liquid and gas-liquid-solid flow systems using electrical capacitance tomography (ECT), Chem. Eng. Sci. 56 (2001) 6455-6462.

[2] W. Q. Yang and L. H. Peng, Image reconstruction algorithms for electrical capacitance tomography, Meas. Sci. Technol. 14 (2003) R1-R13.

[3] Z. H. Guo, F. Q. Shao and D. Lv, Sensitivity matrix construction for electrical capacitance tomography based on the different model, Flow Meas. Instrum. 20 (2009) 135-144.

[4] Y. S. Kim, S. H. Lee, U. Z. Ijaz, K. Y. Kim and B. Y. Choi, Sensitivity map generation in electrical capacitance tomography using mixed normalization models, Meas. Sci. Technol. 18 (2007) 2092-2102.

[5] D. Y. Dong and S. Q. Guo, Image reconstruction method for electrical capacitance tomography based on the combined series and parallel normalization model, Meas. Sci. Technol. 19 (2008) 045502(8pp).

[6] H. X. Wang and L. F. Zhang, Identification of two-phase flow regimes based on support vector machine and electrical capacitance tomography, Meas. Sci. Technol. 20 (2009) 114007(8pp). 